\title{
A COLLABORATIVE APPROACH TO TEACH MODELING AND CONTROL OF SMART ACTUATORS IN THE MECHANICAL ENGINEERING CURRICULUM
}

\author{
Kam K. Leang ${ }^{\dagger}$ and Gina Pannozzo \\ †Dept. of Mechanical Engineering \\ †Dept. of Foundations in Education \\ Virginia Commonwealth University \\ Richmond, VA 23284 \\ Email: \{kkleang, gmpannozzo\}@vcu.edu
}

\author{
Qingze Zou \\ Dept. of Mechanical Engineering \\ lowa State University \\ Ames, IA 50011 \\ Email: qzzou@iastate.edu
}

\author{
Santosh Devasia \\ Dept. of Mechanical Engineering \\ University of Washington \\ Seattle, WA 98195 \\ Email: sdevasia@u.washington.edu
}

\section{ABSTRACT}

In this article, we describe a collaborative approach to develop, integrate, and assess a teaching module on smart actuators specifically designed to embed topics in nano/bio technology into the undergraduate mechanical engineering $(M E)$ curriculum. The collaboration involves three universities, each focusing on one specific aspect of the module. The module consists of lectures and laboratory activities that cover modeling and control of smart actuators for courses such as system dynamics, controls, and mechatronics. The integration of smart actuators such as piezoelectric, shape memory alloy (SMA), and magnetostrictive based devices - into the ME curriculum is important because these devices are the workhorse in a multitude of nano and bio technologies. Thus, these devices play a critical role in the emerging areas, analogous to the benefits of the electric motor at the macroscale. But contrast to the well established coverage of the electric motor in the ME curriculum, modeling and control of smart actuators has yet to be systematically presented in core ME courses. The contribution of this article is presenting the systematic development, integration, and assessment of a teaching module on smart actuators. We first describe the design of lecture components using the piezo actuator as an example. The lecture materials cover core concepts within the framework of dynamics and controls, such as electromechanical coupling, dynamic response, nonlinear input-output behavior, and PID feedback control technique for high-precision positioning. Afterwards, we describe the development of a hands-on laboratory experiment designed to expose students to the basics of experimental modeling of the piezo actuator. The platform is also suited for basic control applications, and an example is presented to illustrate the application of piezo actuator control for high-precision positioning. The paper concludes with a discussion on how the module will be implemented and assessed at the three participating universities.

\section{INTRODUCTION}

Our nation has identified the need to prepare the future workforce to meet the demands of emerging nano and bio technologies [1]. From a mechanical engineering (ME) education standpoint, this need demands the integration of nano/bio technology in the undergraduate curriculum. Motivated by such a demand, we propose a collaborative approach which involves three universities to develop, integrate, and assess a teaching module on smart actuators for three different courses in the ME undergraduate curriculum: a junior/senior-level course on dynamics systems and controls, a senior-level mechatronics course, and a capstone design course.

Smart actuators - for example the piezoelectric actuator (or piezo actuator) - are important because of their wide-spread use in a multitude of emerging nano and bio technologies. The subject module will address the important aspects of modeling, control, and design of smart actuator-based systems through a suite of lectures and laboratory teaching materials. The learning exercises aim to equip students with the knowledge, understanding, and skills necessary for tackling problems in nano/biorelated professional fields. 
Preparing the workforce with the skills to succeed in nano/bio technology is essential to maintaining U.S. economic leadership [2]. Recent trends strongly suggest that these emerging technologies will lead to a technological revolution that will profoundly impact all sectors of the economy (as well as society) [3]. It is projected that over 7 million jobs will be created in these fields world wide by 2015 [4]. This projection is plausible, considering currently the U.S. biomedical technology industry — with its $\$ 78$ billion annual production — demands highly qualified (and a large number of) engineers from traditional engineering specialties such as ME [5]. The demand to properly prepare the future workforce has raised interest by the ME academic community that nano/bio technology should be one of the new "thrust areas" in ME education [6]. Therefore, the need to integrate nano/bio technology into the ME curriculum motivates this project.

Providing students with the knowledge of, and hands-on design experience with, smart actuators is one crucial step in integrating nano/bio technology into ME education. As noted, the study of smart actuators - such as piezoelectric, shape memory alloy (SMA), and magnetostrictive based devices - is important because they are heavily used in a multitude of nano and bio technologies. For example, piezo actuators are used for nanopositioning in atomic force microscopy (AFM) to image and manipulate samples at the nanoscale [7] - AFM is one of the most important instruments in nanotechnology; and SMA actuators are used in micro-pumps [8] and minimum invasive surgical tools in biomedical applications [9]. Thus, smart actuators play a critical role in these emerging areas, similar to the role that electric motors have in industry and society. But contrast to the well-established coverage of electric motors in the ME curriculum, modeling and control of smart actuators has not yet been systematically presented in core ME courses.

The impact that smart actuators will have is analogous to the impact made by the electric motor since the industrial revolution. Electric motors have revolutionized the productivity of the workplace and contributed significantly to bring society to its current state $[10,11]$. Such advancement, however, would not have been possible without encompassing/embracing the fundamentals of modeling/control of electric drives in the engineering curriculum. For instance, electric motors are covered in the widely used ME texts $[12,13]$ and the subject is tightly woven into core ME classes such as system dynamics and mechatronics. As nano/bio technology begins to unfold, smart actuators will play a key role in revolutionizing the productivity of emerging nano and bio applications. There's no question about the impact of these emerging technologies, but to ensure their advancement, it is important to properly prepare the future workforce for these areas.

Mechanical engineering programs around the country have developed nanotechnology-related undergraduate courses, for example, the "Nano-to-Macro Transport Processes" course at MIT, the "Nanomanufacturing and MEMS Technology" (ME
528) course at ISU, the "Micro/Nano Science and Engineering" (ME 495) course at Northwestern University, and the "Micro/Nano-Robotics" (ME 24779-A) course at Carnegie Mellon University (CMU). These courses address critical issues in nanotechnology-related fields, such as manufacturing, design, and control. In contrast, we consider developing a module over creating an entirely new course. This approach has two advantages over the new-course approach used at other schools. First, it is better to integrate concepts all through the curriculum, especially in core courses, rather than in one course [14]. Second, it is difficult to introduce new core courses into an existing curriculum. (The ABET criteria [14] focuses on the learning objectives and outcomes of core courses because core courses impact a larger number of students, compared to, for example, technical elective courses). Technical electives courses are typically easier to create and offer to students, but not all students take these courses. Therefore, the proposed approach enables the integration of the subject concepts into the core curriculum. In this sense a module can be more easily integrated into the curriculum at other schools.

To take a lead in exposing students to the fundamentals and importance of smart actuators, we exploit a collaboration between three universities to develop, integrate, and assess a teaching module that focuses on the modeling, characterization, and control of smart actuators. The piezo actuator, a common smart actuator, is used as an example. The module is created based on our research expertise with developing advanced modeling and control techniques for smart actuators, e.g., [15-19], and in essence, this work integrates research with education. The contribution of this paper is it presents the initial planning phase of the collaborative efforts to develop the piezo actuator module. The module consists of lectures (theory) and laboratory (experiment) activities to address three important aspects: (1) physics, modeling and characterization, (2) control, and (3) design. We will also discuss the implementation and methods by which the effectiveness of the module will be assessed.

The remainder of this paper is organized as follows. In Section 2, we discuss the expected learning objectives and outcomes of the module. Afterwards, we describe in detail the lecture and laboratory components related to modeling and control in Sections 3 and 4, respectively; followed by a discussion of the design component in Section 5. The implementation and assessment process are described in Sections 6 and 7. Finally, we present our conclusions in Section 8, followed by acknowledgements in Section 9.

\section{LEARNING OBJECTIVES AND OUTCOMES}

The module development plan begins by identifying the expected learning objectives and outcomes. Upon completion of the piezo actuator module, students should be able to: (a) understand the fundamental concepts of modeling/control of piezo 
actuators, (b) transfer the knowledge to solve engineering problems at higher levels of abstraction, (c) recognize mechanical engineering concepts in nano/bio technologies, and (d) perform experiments. Specifically, the module will emphasize the following topics:

1. Physics, Modeling, and Characterization of Piezo Actuators - students should be able to (1a) understand the piezoelectric affect and constitutive relations, (1b) recognize and model the vibrational dynamics and hysteresis effect, (1c) characterize the hysteresis behavior, and (1d) design experiments to measure the input-output behavior of piezo actuators;

2. Control of Piezo Actuators - students should be able to: (2a) understand the concepts of feedforward and feedback control in the context of piezo actuators, (2b) identify the key issues in designing feedback controllers for piezo actuators, (2c) design a feedforward and feedback controller to compensate for creep, hysteresis and vibration effects, (2d) implement a controller on an experimental system; and

3. Design with Piezo Actuators - students should be able to: (3a) identify problems in the nano/bio fields which can be addressed by applying engineering concepts, (3b) apply engineering concepts to design a piezo-based system for nano/bio technologies, and (3c) construct a prototype system involving a piezo actuator.

To ensure these topics are addressed, we will describe next the lectures and laboratory materials. A partnership between three universities - Iowa State University (ISU), the University of Washington (UW), and Virginia Commonwealth University (VCU) - was formed to collaborate in developing, implementing, and assessing each component of the example piezo actuator module.

\section{PHYSICS, MODELING, AND CHARACTERIZATION OF PIEZO ACTUATORS}

Piezo actuators are used to position a tool relative to a sample surface with sub-nanometer resolution. To teach the fundamentals of piezo actuators, this component consists of two concepts: the piezoelectric effect and dynamic behavior of a piezo actuator.

\subsection{The Piezoelectric Effect and Constitutive Equa- tions}

The objective of this component is to teach students the piezoelectric effect, the fundamental mechanism behind the extraordinary ability for piezoelectric materials to behave as an actuator and a sensor [20]. To illustrate and convey this concept, we use the quartz crystal model to explain the piezoelectric effect: stress induces an electric dipole moment, and likewise, applied voltage causes the crystal structure to deform, as depicted (a)

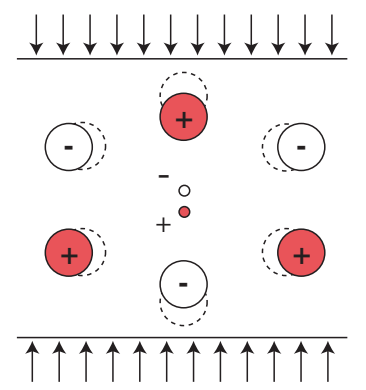

(b)

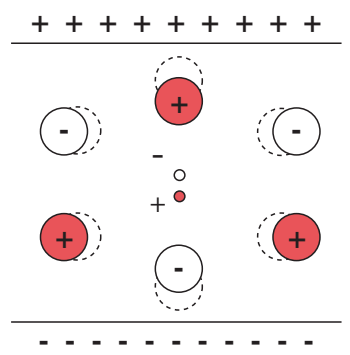

Figure 1. (a) The piezoelectric effect. Stress causes deformation of the crystal lattice, thereby creating an electric dipole. (b) Conversely, an applied electric field causes the lattice to deform accordingly [21].

in Fig. 1(a), (b) [21]. When this model is introduced to students, it's linked to their background in physics and material science courses (e.g., the behavior of charged particles and the crystalline structure of materials such as ceramics [22]). This model gives students a conceptual framework for how a piezo actuator works.

Next, the basic constitutive relationship between the applied input voltage and the resulting displacement of the piezo actuator is taught [23]. The relationship describes the linear electromechanical properties of the device, and it is based on the assumption that the total strain is the sum of mechanical strain induced by mechanical stress and the strain caused by an applied input voltage. Students are asked to verify the relationships through a laboratory exercise (see next section) where they will apply an input voltage and measure the displacement of an experimental piezo actuator.

\subsection{Modeling and Characterization}

The objective of this component is to teach the fundamentals of system dynamics and nonlinear hysteresis effect. The behavior of piezo actuators expresses the combined effects of creep, hysteresis, and vibration [24, 25]. The lectures and laboratory experiments are designed to teach students how to model the linear dynamics (creep and vibration) and the nonlinear hysteresis. Students are also exposed to hands-on experiments to further explore these issues.

Lecture: Modeling Linear Vibrational Dynamics The simplified linear models to capture the creep and vibration effects in piezos are shown in Fig. 2(a) and (b), respectively [25,26]. These models utilize the three common mechanical elements traditionally taught in core ME course on system dynamics (i.e., spring, mass, and damper) to capture the creep and vibration effects. Vibration modes of mechanical systems, a key concept in ME undergraduate dynamics/control courses [27], is integrated to the lecture to facilitate the discussion.

The creep and vibrational effects are modeled using the

$$
\text { Copyright (c) } 2007 \text { by ASME }
$$




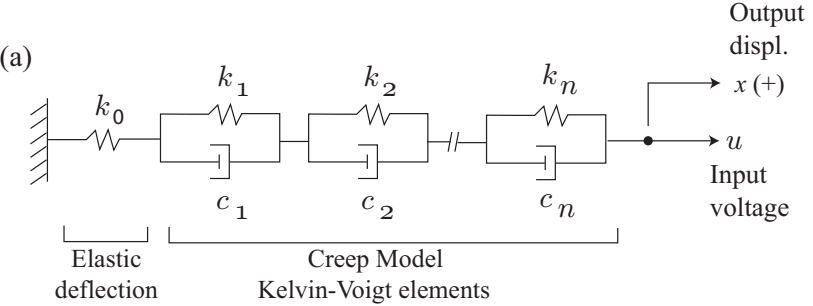

(b)

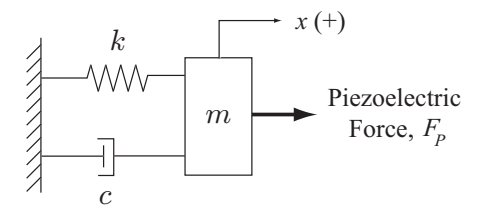

Figure 2. Modeling the dynamic behavior of a piezo actuator: (a) creep model and (b) a one-dimensional vibrational dynamics model.

transfer function concept $[25,28]$. For example as shown in Fig. 2(a), an input voltage $u$ causes a displacement $x$ of the creep model which gives

$$
G_{\text {creep }}(s) \triangleq \frac{x(s)}{u(s)}=\frac{1}{k_{0}}+\sum_{i=1}^{N} \frac{1}{s c_{i}+k_{i}},
$$

where $k_{i}$ and $c_{i}$ are the spring and damper constants, respectively. Similarly, by assuming the piezoelectric force $F_{p}$ is proportional to the applied input voltage (i.e., $F_{p}=\alpha u$ ), the vibrational-effect in the piezo actuator's input-output response can be effectively captured by the following second-order transfer function model:

$$
\begin{aligned}
G_{\text {vibration }}(s) \triangleq \frac{x(s)}{u(s)} & =\bar{K} \frac{\omega_{n}^{2}}{s^{2}+2 \zeta \omega_{n} s+\omega_{n}^{2}} \\
& =\frac{\alpha / m}{s^{2}+(c / m) s+k / m} .
\end{aligned}
$$

Therefore, the key concepts pertaining to first and second-order dynamic systems [27], as conventionally taught in ME dynamics/control courses, applies equally to the example piezo actuator.

By using MATLAB software, students are asked to investigate the properties of these models due to various input voltages, for instance, an impulse input, a step input, a ramp, as well as sinusoidal and triangular trajectories. Additionally, they can study the effects of exciting the resonant mode by applying relatively-high frequency triangle inputs - these inputs induce oscillations and the oscillations limit the precision and operating speed of piezo-based systems in nano/bio technology [25]. Moreover, understanding this behavior is critical in the design of nano-precision positioning systems, for applications such as scanning probe microscopes (SPMs).

Experiment: Linear Vibrational Dynamics The objective of the laboratory experiments is to give students hands-on skills

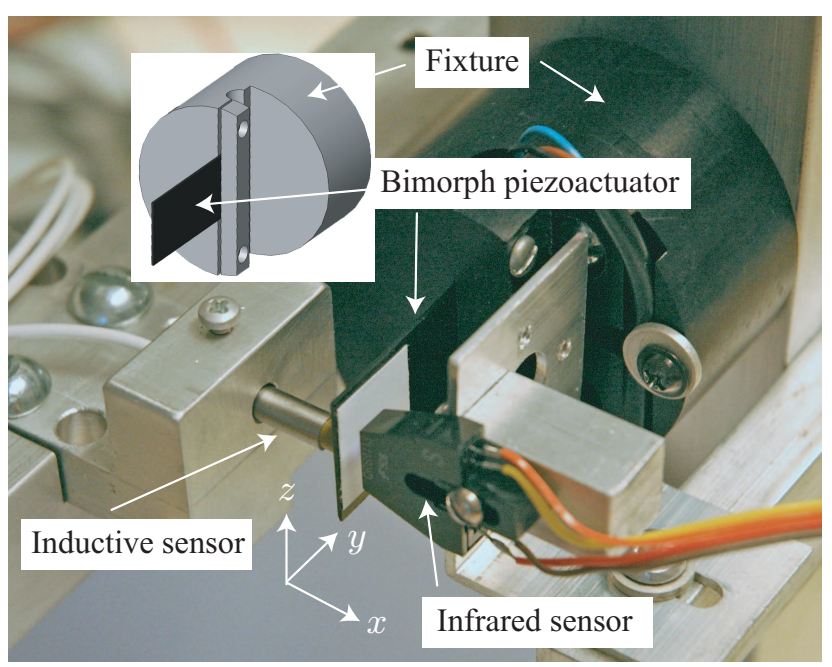

Figure 3. A modular piezo actuator experiment for laboratory activities.

[14] with observing, measuring, and controlling the behavior of piezo actuators. A modular experimental system shown in Fig. 3 was developed for the laboratory activities. It consists of a bimorph piezo actuator, an inductive displacement sensor, a lowcost infrared sensor, a high-voltage amplifier (not shown) to drive the piezo actuator, and a PC with a data acquisition card (not shown). The measurement and control software can be written in MATLAB (xPC-Target), LabView, or C-programming. Students are given the task of calibrating the sensors.

This laboratory exercise exposes students to the basics of measuring input-output responses of a dynamic system and obtaining a dynamics model from the measured output response. First, students are instructed to design a simple data acquisition program to observe and measure the vibrational dynamics effect of the piezo actuator (either by coding in MATLAB xPC-Target, LabView, or C). For example, the program sends a triangle waveform at various frequencies, and then measures the output displacement of the piezo actuator. Then the vibrational dynamics effect can be observed by comparing the applied input with the measured output, for instance as shown in Fig. 4(a). In Fig. 4(a), the oscillations in the output response (displacement) is caused by the vibrational dynamics, and the magnitude of the oscillations depends on the frequency of the input signal. Then, the exercise asks students to measure the frequency response of the piezo actuator using a dynamic signal analyzer (DSA). The expected frequency response is shown in Fig. 4 (b) and (c). Finally, students determine a transfer function model of the vibrational dynamics by curve-fitting the measured frequency response using MATLAB software [29]. Additionally, they are asked to validate the transfer function model by applying a step input to the piezo actuator, then to compare the measured response with the model's response. 
(a)

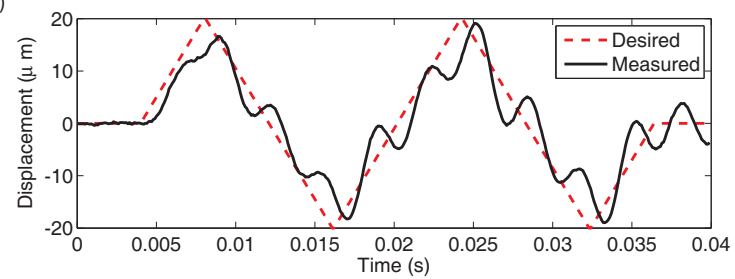

(b)
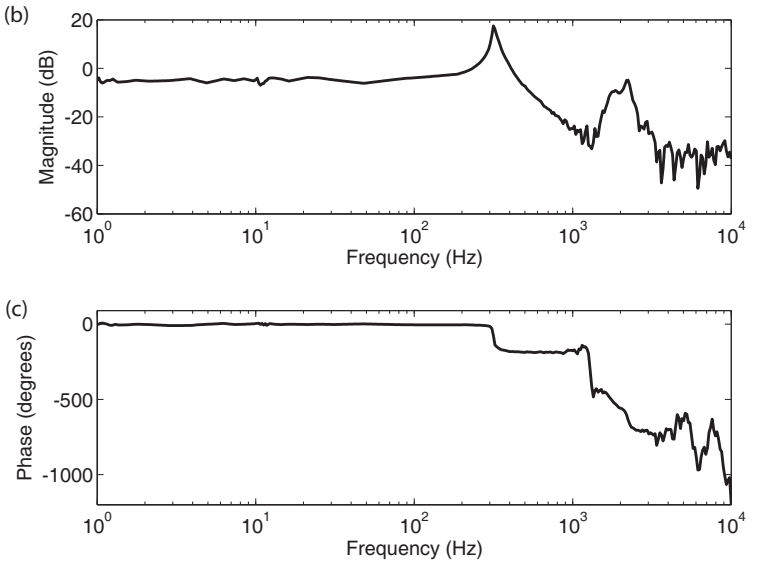

Figure 4. Effect of vibration: (a) piezo actuator output response due to $50 \mathrm{~Hz}$ triangle input voltage, and (b) magnitude and (c) phase versus frequency response for the experimental piezo actuator shown in Fig. 3.

Lecture: Nonlinear Hysteresis Hysteresis effect becomes significant when piezo actuators displace over relatively large range $[30,31]$. This effect causes loss in precision positioning, for instance in AFM systems [19,32]. This lecture conveys the basic mechanisms of hysteresis (the energy loss caused by micro-scale domain wall interactions) [33], and the properties of hysteresis (e.g., the local memory property) [34]. Furthermore, students learn various methods to quantify and characterize the hysteresis behavior.

Experiment: Nonlinear Hysteresis To help students gain hands-on experience with hysteresis effect, they are asked to observe and model this behavior. The exercise calls for students to apply a triangle input signal of various amplitudes to the piezo actuator and compare the input and output signals (see example result in Fig. 5). The various amplitudes are chosen because hysteresis can be regarded as a range-dependent distortion [25]. From these measurements, students are asked to characterize the amount of hysteresis (i.e., distortion) present in the piezo actuator. Then, they use the input-output data (e.g., Fig. 5(a) and (b)) to construct a simplified input-output model based on a look-up table, i.e., they correlate each input value with an output value (see Fig. 5(c)). Such models can be used to design controllers to compensate for hysteresis $[31,35,36]$.
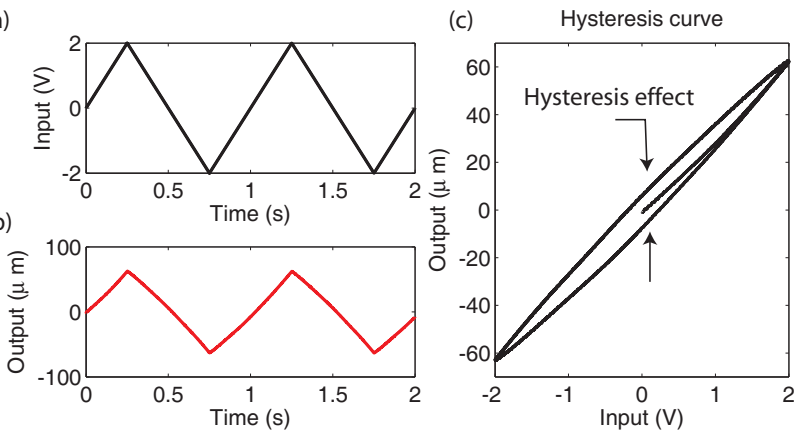

Figure 5. Hysteresis effect: (a) applied input voltage vs. time, (b) measured output response vs. time, and (c) hysteresis curve (output vs. input).

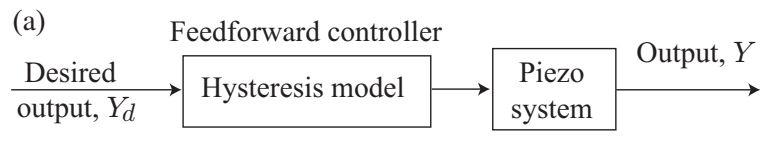

(b)

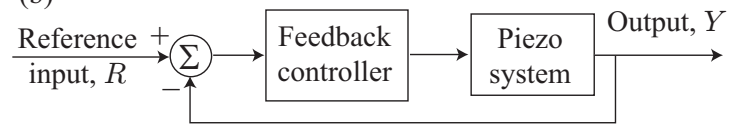

Figure 6. Block diagram of (a) feedforward and (b) feedback approach to control a piezo actuator.

\section{PRECISION CONTROL OF PIEZO ACTUATORS}

This component focuses on teaching students the fundamental control techniques for high-precision positioning of piezo actuators. They are taught the foundations of feedforward $[25,35,36]$ and feedback control techniques [32, 37, 38] for piezoactuators (see control schemes shown in Fig. 6). The lecture module on characterization and modeling of piezoactuators, as described in Section 3, is presented a priori to facilitate the learning of these concepts.

Lecture: Feedforward Control The feedforward technique is the process by which a model is exploited to control a system. The feedforward input can be found to compensate for positioning errors due to creep, vibration, and hysteresis by inverting a model, for example a vibrational dynamics or a hysteresis inputoutput model $[25,35,36]$. This lecture adopts a simple method involving a look-up table to implement the feedforward controller, where the table is constructed from the measured input-output behavior, for example, by experimentally measuring the hysteresis curve (see previous section). Other basic feedforward approaches to directly control the piezoactuator are also covered in this lecture [25].

Experiment: Feedforward Control This exercise gives students the opportunity to design and implement a simplified feed- 
forward controller to compensate for the hysteresis behavior. The experiment utilizes the experimental piezo system as shown in Fig. 3. First, students are asked to experimentally obtain an input-output plot of the hysteresis behavior (see modeling component above). Then, they use the data to construct a simple look-up table model to describe the hysteresis. Afterwards, they invert the model to find the feedforward input to compensate for hysteresis effect (see Fig. 6). Then students analyze their design using MATLAB simulations, and finally apply their design to the experimental system. Students are required to measure the output response and quantify the performance of their design. Additionally, they also discuss implementation issues and the limitations of their designs.

Lecture: Feedback Control This component teaches the fundamentals of feedback control for precision positioning of piezoactuators (see Fig. 6(b)). Feedback control, for example, proportional-integral-derivative (PID), is widely used in industry to control piezoactuators in SPM-based systems [32, 37, 38]. The lecture material covers the issues of designing PID feedback controller (for piezo actuators) with low gain margin — the low gain margin is typical of piezoactuators and it can lead to the instability of the feedback system [15]. The concept of high-gain feedback [26] will be introduced to account for the hysteresis and creep behavior, as well as to improve the transient response and the bandwidth of the positioning system. We note that PID controller design is standard in the undergraduate ME curriculum [39]), thus this lecture component can be seamlessly integrated into existing ME courses with the advantage of introducing new application involving piezoactuators.

Experiment: Feedback Control Students are asked to design a PID controller for an experimental piezoactuator to achieve a desired response. They begin by simulating the controller in MATLAB software, then applying their design to control the experimental piezo-positioning system (Fig. 3). Key control-design concepts such as percent overshoot, settling time, and steadystate error [39] are also discussed. Students are asked to use these concepts to quantify the performance of their controller design.

The lecture and laboratory components described above teach core concepts in system dynamics, control, and mechatronics. As a result, each component can easily and seamlessly be integrated to existing ME courses. Not only are the core concepts in modeling, characterization, and control covered by the proposed module, but the module also introduces students to the new topic of piezo-based nanopositioning.

\section{DESIGN COMPONENT}

Offering educational experiences that resemble what people need to do in the workplace prepares students for future tasks in non-school settings [40]. Engineering students are typically pro- vided with such an open-ended real-world design experience in the capstone project (as part of design experience requirements of ABET [14]). Therefore, this component exposes students to the design experience, as well as with design issues related to piezoactuators to prepare them for future work at the interface between engineering and nano/bio technology. In particular, this component aims to enable abstract representation of problems, for example, biomedical instrumentation involving piezoactuators, so that engineering concepts can be applied to solve them. A team of students is asked to abstract, for example, the problem of high-speed cell imaging with an AFM [15] into engineering requirements on the piezoactuator such as bandwidth, positioning accuracy, and resolution of data acquisition systems. These engineering requirements are used to design (build, control, and test) a high-speed piezoactuator experimental system similar to the setup shown in Fig. 3. Students are also required to perform an evaluation of their design to determine the achievable bandwidth and positioning accuracy of the system. Additionally, they provide a cost analysis, manufacturing parameters, sustainability, and health and safety considerations.

\section{IMPLEMENTATION}

Each learning component described above is implemented in three courses - junior and senior level courses - as shown in Fig. 7.

At ISU, ME421, Mechanical Systems and Control, is one of the 14 core ME undergraduate courses. The course focuses on introducing students to the art and science of modeling and analysis, simulation, and control of dynamic systems. Additionally, the course provides students the opportunity to experimentally implement closed-loop control schemes. Currently, concepts on modeling/control of electric motors are extensively discussed in ME421. As previously mentioned, these core concepts can also be taught in the context of piezoactuators. Particularly, the modeling aspects of the piezoactuator module is targeted for this course.

At VCU, EGRM 410 Mechatronics is a core senior-level course. The focus of the course is to teach the fundamental concepts of mechatronics, which is the integration of mechanical, electrical, and computer systems in design. Students are exposed to both theory and experiment materials which cover various concepts related to mechatronic systems [41]. In particular, control of electro-mechanical systems, such as DC motors, is one main component of the course - e.g., students build circuits to control DC motor, servo motors, stepper motors, and they design feedback controllers for simplified servo-systems. The aspect of control issues of piezoactuators is implemented in this course to expose students to engineering concepts of smart actuators, the analogy of the electric motor in the micro- to nano-scale world.

Finally, ME495M at UW is a Senior-level Capstone Design Course where students work in multidisciplinary teams on an 


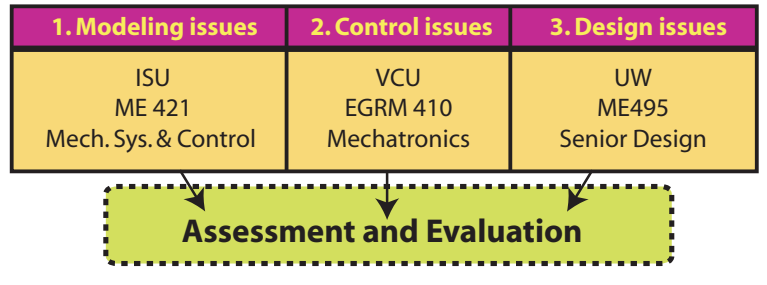

Figure 7. The implementation and assessment layout for the piezoactuator module.

open-ended design project as part of the core ME requirements. Traditional issues in design are addressed in this course through open-ended projects. Hence this course is well-suited for implementing the design aspect of the piezoactuator module.

\section{ASSESSMENT}

The goal of the assessment plan is to systematically examine the impact of the developed module and to determine whether the learning outcomes have been achieved through the use of both formative and summative evaluation procedures, and qualitative and quantitative information. The assessment and evaluation investigates students' learning and knowledge, students' attitudes and perceptions, and faculty development. The data facilitates the understanding of the project impact from design to implementation, and the interpretation of final outcomes $[42,43]$. Formative evaluation procedures provides immediate feedback in an on-going assessment and evaluation process to aid continuous modification (both content and instruction) [44] throughout the project. For example, general ME content knowledge and specific knowledge of modeling, control, and design with piezoactuators are assessed using a pre-test at the start of each semester/quarter when the module is implemented. The results of the pre-test are used as a baseline to guide instructional planning and to compare the levels of students' a priori knowledge across the three institutions. Furthermore, quantitative data assessing content knowledge is collected through homework assignments, quizzes, course projects, and mid-term exam. Each of these consists of a combination of both selectedand constructed-response item formats that will vary according to the course content and objectives. Including both selectedand constructed-response formats allows for assessment of both wider range of learning objectives to evaluate the breadth and depth of technical understanding of concepts at lower and higher levels of cognitive processing [45]. The ability of students to transfer knowledge is assessed through specially designed homework assignments, quizzes, course projects, and exams. Student attitudes and perceptions are assessed at pre-test and then periodically using both formal and informal surveys. Results of the informal and formal surveys are used to enhance instruction and teaching of the modules. Student cognitive learning style -
Students' cognitive learning style is assessed at pre-test using a widely used measure of cognitive learning style, e.g., [46].

Summative assessment is conducted to measure student learning through a cumulative final exam in each course. Students' knowledge, reasoning skills, and ability to transfer knowledge are assessed. The evaluation includes a combination of both selected- and constructed-response item formats that varies according to the course content and objectives, and cover incorporate both course-specific and overlapping items to permit both within- and across-institution comparisons. Likewise, student attitudes and perceptions are assessed using the same formal survey developed for the formative assessment. This enables the examination of change in attitudes as a result of the project with a specific focus on the content and format of the modules. In addition, the course evaluations used at each institution are used to provide information regarding students' perceptions of the instruction across the course as a whole.

\section{CONCLUSIONS AND FUTURE WORK}

We described the design of a teaching module to address the need to integrate nano/bio technology into the ME undergraduate curriculum. The piezoactuator was the centerpiece for the module, where lectures and laboratory activities (components) were developed to teach the fundamentals of modeling, control, and design. A collaboration between three universities was formed to develop each component of the module and to implement the teaching materials. We described an assessment plan to evaluate the impact of the module. We plan to develop modules that cover other smart actuators, including SMA, magnetostrictive devices, and electro-active polymers; and utilize the assessment results from implementing the piezoactuator module to improve all aspects of the module (modeling, control, and design) with the objective of integrating it into all levels of the ME curriculum.

\section{ACKNOWLEDGEMENT}

Authors gratefully acknowledge the support of National Science Foundation DUE Grant \#0633098.

\section{REFERENCES}

[1] National Nantotechnology Initiative, 2004. Investment mode 2: NNI grand challenge areas. Tech. rep.

[2] Marberger, J. H., and Kavamme, E. F., 2005. The national nanotechnology initiative at five years: assessment and recommendations of the national nanotechnology advisory panel. Tech. rep., President's Council of Advisors on Science and Technology.

[3] Roco, M. C., 2003. "Broader societal issues of nanotechnology”. J. Nanoparticle Res., 5, pp. 181-189. 
[4] Roco, M. C., and Bainbridge, W. S., 2001. Societal implications of nanoscience and nanotechnology. Tech. rep., National Science Foundation.

[5] U.S. Department of Labor Bureau of Labor Statistics, 2003. Occupational outlook handbook, biomedical engineers, , 2002-03 edition. Tech. rep.

[6] Akay, A., 2002. Report from a workshop organized by the big-ten-plus mechanical engineering department heads. Tech. rep., The National Science Foundation.

[7] Wiesendanger, R., 1994. Scanning probe microscopy and spectroscopy. Cambridge University Press, Cambridge.

[8] Park, J.-H., Yoshida, K., and Yokota, S., 1997. "A piezoelectric micropump using resonance drive: proposal of resonance drive and basic experiments on pump characteristics". In Proc. of the 1997 ASME Inter. Mech. Eng. Congress and Exposition: The Fluid Power and Systems Technology Division, Vol. 4, pp. 77-82.

[9] Stevens, J. M., and Buckner, G. D., 2005. "Actuation and control strategies for miniature robotic surgical systems". ASME Journal of Dynamic Systems, Measurment, and Control, 127, pp. $537-549$.

[10] Boff, R. B. D., 1967. "The introduction of electric power in American manufacturing". Economic History Review, 20(3), pp. $509-518$.

[11] Bunch, B., and Hellemans, A., 2004. The history of science and technology. Houghton Mifflin Company, Boston.

[12] Palm, I. W. J., 2005. System dynamics. McGraw Hill, Boston.

[13] Alciatore, D. G., and Histand, M. B., 2003. Introduction to mechatronics and measurement systems, 2nd edition ed. McGraw Hill, Boston.

[14] ABET, 2004. Engineering accreditation commission: 2005-2006 criteria for accrediting engineering programs.

[15] Zou, Q., Leang, K. K., Sadoun, E., Reed, M. J., and Devasia, S., 2004. "Control issues in high-speed AFM for biological applications: collagen imaging example". Asian Journal of Control, 6(2), pp. 164-178.

[16] Tien, S., Zou, Q., and Devasia, S., 2004. "Preview-based optimal inversion for output tracking: application to scanning tunneling microscopy". IEEE Trans. Contr. Syst. Tech., 12(3), pp. 375 - 386.

[17] Perez, H., Zou, Q., and Devasia, S., 2004. "Design and control of optimal scan trajectories: scanning tunneling microscope example". ASME J. Dyn. Syst. Meas. and Cont., 126(1), pp. 187-197.

[18] Zou, Q., Vander Giessen, C., Garbini, J., and Devasia, S., 2005. "Precision tracking of driving waveforms for inertial reaction devices". Rev. Sci. Instr., 76(1), pp. 23701-23709.

[19] Leang, K. K., and Devasia, S., 2006. "Design of hysteresiscompensating iterative learning control for piezo positioners: application to atomic force microscopes". Mechatronics, 6(3-4), pp. $141-158$.
[20] Cady, W. G., 1946. Piezoelectricity. McGraw-Hill, New York.

[21] Mason, W. P., 1946. "Quartz crystals for electrical circuits". R. A. Heising, ed., D. Van Nostrand Co., Inc., pp. 11-56.

[22] Callister, W. D., 1994. Materials science and engineering: an introduction. John Wiley and Sons, Inc., New York.

[23] of Electrical, I., and Inc., E. E., 1988. IEEE standard on piezoelectricity. Tech. Rep. ANSI/IEEE Std. 176 - 1987.

[24] Jung, H., and Gweon, D.-G., 2000. "Creep characteristics of piezoelectric actuators”. Rev. Sci. Instr., 71(4), pp. 18961900.

[25] Croft, D., Shed, G., and Devasia, S., 2001. "Creep, hysteresis, and vibration compensation for piezoactuators: atomic force microscopy application". ASME J. Dyn. Syst., Meas., and Control, 123, pp. 35-43.

[26] Leang, K. K., and Devasia, S., 2002. "Hysteresis, creep, and vibration compensation for piezoactuators: feedback and feedforward control". In The 2nd IFAC Conference on Mechatronic Systems, pp. 283-289.

[27] Ogata, K., 2004. System dynamics, fourth ed. Prentice Hall.

[28] Malvern, L. E., 1969. Introduction to the mechanics of a continuous medium. Prentice-Hall, Englewood Cliffs, NJ.

[29] Leang, K. K., Zou, Q., and Devasia, S., 2002. "The crc mechatronics handbook". R. Bishop, ed., The CRC Press, pp. 40-54.

[30] Smith, R. C., Seelecke, S., Dapino, M., and Ounaies, Z., 2006. "A unified framework for modeling hysteresis in ferroic materials". Journal of the Mechanics and Physics of Solids, 54, pp. $46-85$.

[31] Ge, P., and Jouaneh, M., 1996. "Tracking control of a piezoceramic actuator". IEEE Trans. Contr. Syst. Tech., 4(3), pp. 209-216.

[32] Barrett, R. C., and Quate, C. F., 1991. "Optical scancorrection system applied to atomic force microscopy". Rev. Sci. Instr., 62(6), pp. 1393-1399.

[33] Jiles, D. C., and Atherton, D. L., 1986. "Theory of ferromagnetic hysteresis". J. Magnetism and Magnetic Materials, 61, pp. 48-60.

[34] Mayergoyz, I. D., 1991. Mathematical models of hysteresis. Springer-Verlag, New York.

[35] Jorgensen, J. F., Carneiro, K., Madsen, L. L., and Conradsen, K., 1994. "Hysteresis correction of scanning tunneling microscope images". J. Vac. Sci. Technol. B, 12(3), pp. 1702-1704.

[36] Dirscherl, K., Garnaes, J., Nielsen, L., Jogensen, J. F., and Sorensen, M. P., 2000. "Modeling the hysteresis of a scanning probe microscope". J. Vac. Sci. Technol. B, 18(2), pp. 621-625.

[37] Daniele, A., Salapaka, S., Salapaka, M. V., and Dahleh, M., 1999. "Piezoelectric scanners for atomic force microscopes: design of lateral sensors, identification and control". In Proc. American Control Conference, pp. 253-257. 
[38] Salapaka, S., Sebastin, A., Cleveland, J. P., and Salapaka, M. V., 2002. "High bandwidth nano-positioner: a robust control approach". Rev. Sci. Instr., 73(9), pp. 3232-3241.

[39] Dorf, R. C., and Bishop, R. H., 2001. Modern control systems. Prentice-Hall, Upper Saddle River.

[40] Bransford, J. D., Brown, A. L., and Cocking, R. R., 2000. How people learn: brain, mind, experience, and school expanded edition. Committee on Developments in the Science of Learning with additional material from the Committee on Learning Research and Educational Practice, National Academy Press, Washington, D.C.

[41] Ashley, S., 1997. "Getting a hold of mechatronics". ASME Mechanical Engineering Magazine, 119(5), pp. 60 - 63.

[42] Rossi, P. H., Lipsey, M. W., and Freeman, H. E., 2004. Evaluation: a systematic approach, 7th edition ed. SAGE Publications, Thousand Oaks, CA.

[43] Sundberg, M. D., 2002. "Assessing student learning”. Cell Biology Education, 1, pp. $11-15$.

[44] Frechtling, J., Westat, Frierson, H., Hood, S., and Hughes, G., 2002. The 2002 user friendly handbook for project evaluation. Tech. Rep. NSF 93-152, National Science Foundation, Washington, DC.

[45] Popham, W. J., 2000. Modern educational measurement: practical guidelines for educational leaders. Allyn and Bacon, Boston.

[46] Felder, R. M., and Spurlin, J., 2005. "Applications, reliability and validity of the index of learning styles". Int. J. Engng. Ed., 21(1), pp. 103-112. 